\title{
Growth and Characterisation of Nonlinear Optical Single Crystals of L-Alaninium Oxalate
}

\author{
K. J. Arun ${ }^{*}$ and S. Jayalekshmi \\ Division for Research in Advanced Materials, Department of Physics, \\ Cochin University of Science and Technology, Kochi-682022, Kerala, India. \\ *Corresponding author: arun_k_j@yahoo.co.in,jayalekshmi@cusat.ac.in
}

\begin{abstract}
A potential organic nonlinear optical (NLO) single crystal based on amino acid, $L$ alaninium oxalate (LAO) is grown using the simple chiral amino acid L alanine and oxalic acid by slow evaporation method. Grown crystals have an optimum dimension of $40 \times 15 \times 8 \mathrm{~mm}^{3}$ with good optical quality and are characterized by powder XRD, CHN analysis, FTIR spectroscopy, TGADTA, DSC, UV/VIS/ NIR absorption spectroscopy, photoconductivity and photoluminescence studies. The third order nonlinear optical properties of the grown crystals are investigated employing open aperture Z scan technique.
\end{abstract}

Key words: Nonlinear optical crystal, photoconductivity, photoluminescence, Z scan.

\section{INTRODUCTION}

Over the past two decades much attention has been paid to the search of novel good quality NLO materials that can generate large second order optical non linearities, significant for potential applications including telecommunication, optical computing, and optical data storage and processing[1 - 6]. In many of the organic NLO materials there is a solid framework of conjugated $\pi$ electrons along with weak Van der Waals and hydrogen bonds which are responsible for most of their NLO properties. Organic NLO materials are superior to their 
inorganic counterparts due to high conversion efficiency for second harmonic generation and good transparency in the visible region, high resistance to optical damage and so on.

The $\alpha$ amino acid $\mathrm{L}$ alanine can be considered as the fundamental building block of more complex amino acids which shows strong non linear behaviour and anomalous phonon coupling and is a system exhibiting vibrational solitons [7]. An attempt is made to synthesize and grow an organic NLO material L alaninium oxalate (LAO) using L alanine and oxalic acid. The top seeded, aqueous solution grown crystals of LAO by slow evaporation are characterized by powder XRD, TGA- DTA, DSC, FTIR,CHN analysis, UV/Vis/NIR absorption spectroscopy, and photoconductivity and photoluminescence studies. Nonlinear absorption in the sample is established by open aperture $\mathrm{z}$ scan technique.

\section{SYNTHESIS AND GROWTH}

$\mathrm{L}$ alaninium oxalate is synthesized from equimolar solution of $\mathrm{L}$ alanine and oxalic acid by evaporation preventing decomposition. Synthesized samples are crystallized repeatedly to get a pure, colorless, crystalline powder. Good quality seed crystals are prepared using this powder. One or two seed crystals are then placed in its saturated solution kept in a bath at $30^{\circ} \mathrm{C}$. Seed crystals are grown to big crystals by slow evaporation to a size of about $40 \times 15 \times 8 \mathrm{~mm}^{3}$ with good transparency in three weeks time (Fig. 1). To the best of our knowledge, growth of big LAO crystals of this size is not reported elsewhere [8].

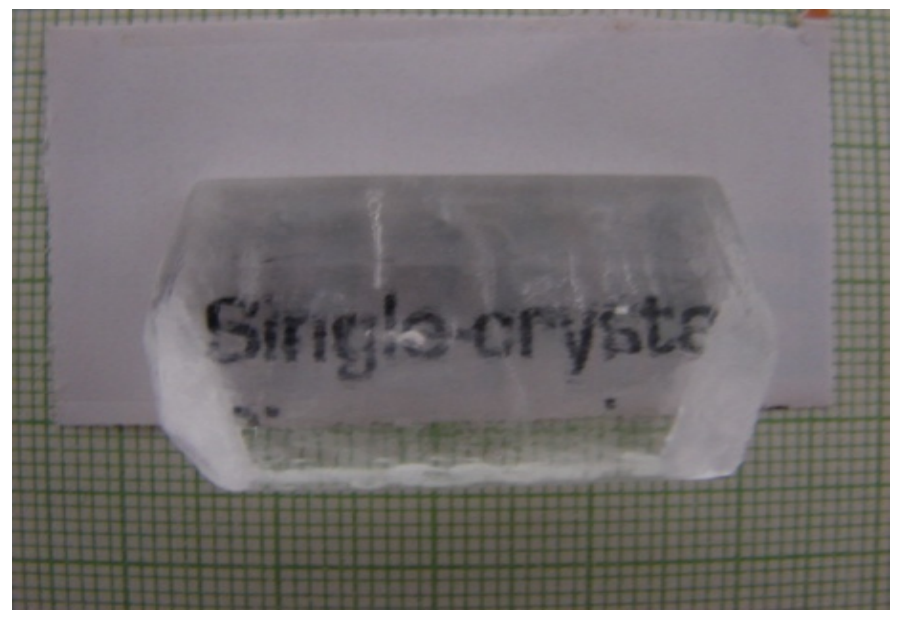

Fig. 1. L-alaninium oxalate single crystal 


\section{CHARECTERISATION METHODS}

Powdered XRD spectrum of the crystals is recorded using Rigaku X ray diffractometer with $\mathrm{CuK} \alpha$ radiation to identify the compound and structure. FTIR spectrum of the crystals is taken with AVTAR 370 spectrometer having DTGS $\mathrm{KBr}$ detector with a resolution of $4 \mathrm{~cm}^{-1}$. TGADTA studies are carried out with Perkin Elmer Diamond TGA / DTA equipment in nitrogen atmosphere at a heating rate of $10^{\circ} \mathrm{C} / \mathrm{min}$ for a range $28-600^{\circ} \mathrm{C}$ to assess the thermal stability. The DSC spectrum of the sample is also recorded with a Mettler Toledo DSC822 instruments with a heating rate of $10^{\circ} \mathrm{C} / \mathrm{min}$. CHN analysis is done with VarioEL III CHNS serial number11035060 to confirm the molecular formula of the crystal. UV/Vis/NIR absorption spectrum is recorded using a JASCO V 570 spectrophotometer in the range from 190nm to $2500 \mathrm{~nm}$. Negative photoconductivity is established using a KEITHLEY 236 source measuring unit. Photo-luminescence spectrum is recorded with a Jobin Yvon Spectrofluorometer (Model FL3-22). A $450 \mathrm{~W}$ xenon lamp is used as the source and PMT (Model R928P) as the detector. Double gratings are used for the excitation and emission spectrometers. $Z$ scan technique is used to reveal the nonlinear optical absorption behaviour of the crystal.

\section{RESULTS AND DISCUSSIONS}

\subsection{X-ray Diffraction Analysis}

From the XRD data, it is found that L-alaninium oxalate belongs to the orthorhombic system with $\mathrm{a}=5.6302 \mathrm{~A}^{\circ}, \mathrm{b}=7.235 \mathrm{~A}^{\circ}$ and $\mathrm{c}=19.5973 \mathrm{~A}^{\circ}$, the space group being $\mathrm{P} 22_{1} 2_{1} 2_{1}$ and has four molecules in the unit cell with a volume of $803.146 \mathrm{~A}^{\circ}$. The close agreement with the observed, calculated and reported $d$ values [9] confirm the identity of the grown crystal. The recorded powder XRD pattern is given in Figure 2. In the layered crystal structure of LAO (Fig. 3), the amino group of the L-alaninium cation forms three $\mathrm{N}-\mathrm{H}$... O hydrogen bonds with the oxygen of semi oxalate group and a symmetry-related alanine cation. There also exists two $\mathrm{O}-\mathrm{H}$...O hydrogen bonds. The alaninium and semi-oxalate ions form alternate columns leading to a layered arrangement parallel to the a.c. plane and each such layer is interconnected to the other through $\mathrm{N}-\mathrm{H} . . . \mathrm{O}$ hydrogen bonds. Two short $\mathrm{C}$... O contacts involving the carboxyl oxygen of the alaninium ion $[\mathrm{C} 1 \ldots \mathrm{O} 2(1 / 2+x, 3 / 2-y,-z)=2.931(3) \AA$ and $\mathrm{C} 2 \ldots \mathrm{O} 2(-1 / 2+x, 3 / 2-y,-$ z) $=2.977(3) \AA]$ are also observed in these layers. The slight difference observed in the bond lengths of C5- O5 $[=1.219(2) \AA]$ and C5-O6 [= 1.235(2) $\AA]$ in the carboxylate group of the semi-oxalate ion may be attributed to the difference in strengths of the $\mathrm{N}-\mathrm{H} \ldots \mathrm{O}$ hydrogen bonds in which both $\mathrm{O} 5$ and $\mathrm{O} 6$ are involved. 


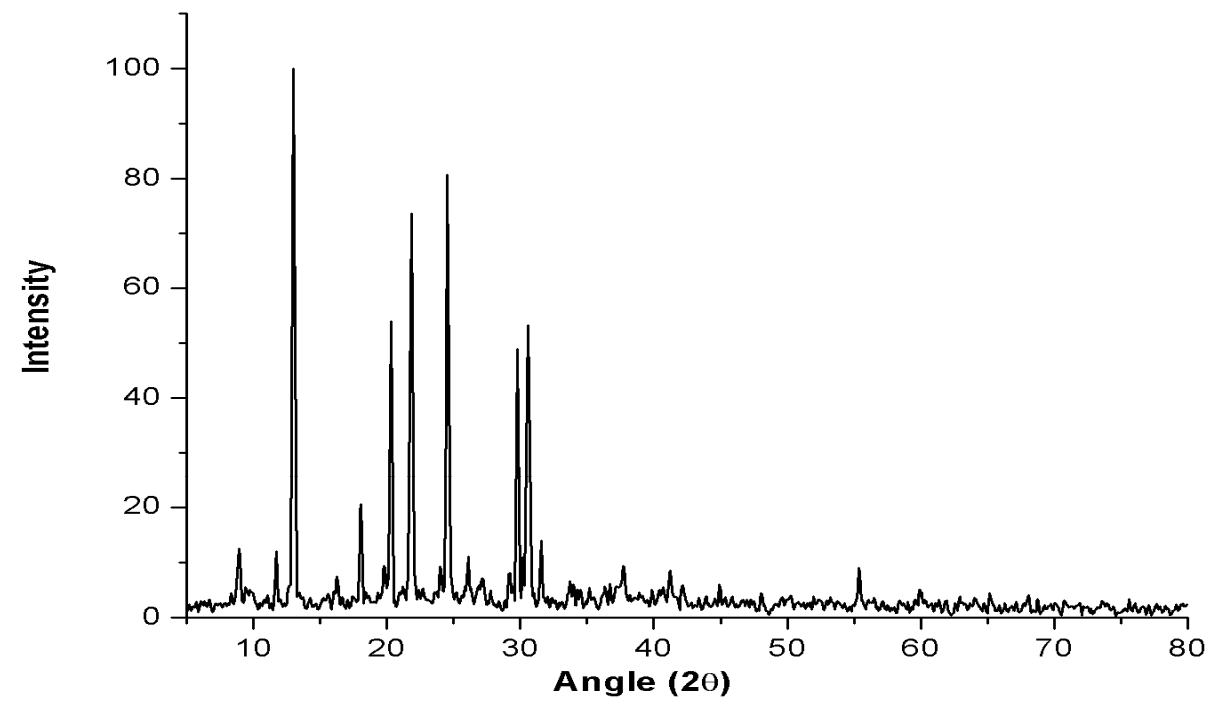

Fig. 2. Powder XRD spectrum of L-alaninium oxalate crystal

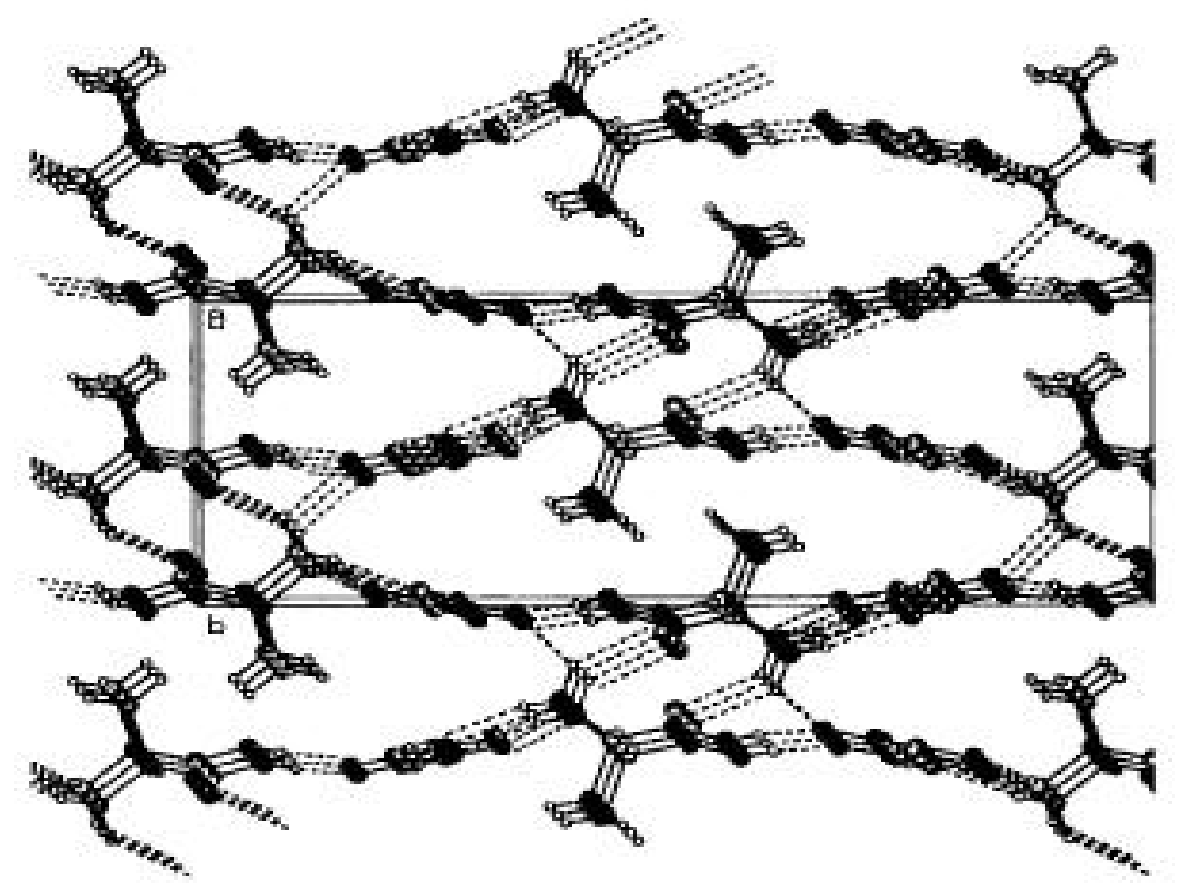

Fig. 3. Layer structure of L-alaninium oxalate 


\subsection{Measurement of Density}

The measurement of density is one of the important methods to study the purity of crystals. The most sensitive method to find the density of the crystal, namely, the swim or sink method [10] is used in the present study. In this analysis, theoretical density value is found using the formula, density $=(M Z) /(N V)$; where $M$ is the molecular weight, $Z$ is the number of molecules per unit cell, $N$ is the Avogadro number, and $V$ is the volume of the unit cell. The density values are experimental: $1.35 \mathrm{~g} / \mathrm{cm}^{3}$; and theoretical: $1.37 \mathrm{~g} / \mathrm{cm}^{3}$.

\subsection{CHN Analysis}

The chemical composition of the grown crystal determined using $\mathrm{CHN}$ analysis reveals that it contains $33.18 \%$ carbon $(33.51 \%), 4.96 \%$ hydrogen $(5.02 \%), 7.79 \%$ nitrogen $(7.82 \%)$ where the figures in braket represent the theoretical composition. Thus the molecular formula of the compound $\mathrm{L}$ alaninium oxalate is established as $\mathrm{C}_{5} \mathrm{H}_{9} \mathrm{NO}_{6}$ and it does not contain any water of crystallisation in its structure. Figure 4 shows the molecular structure of LAO molecule.

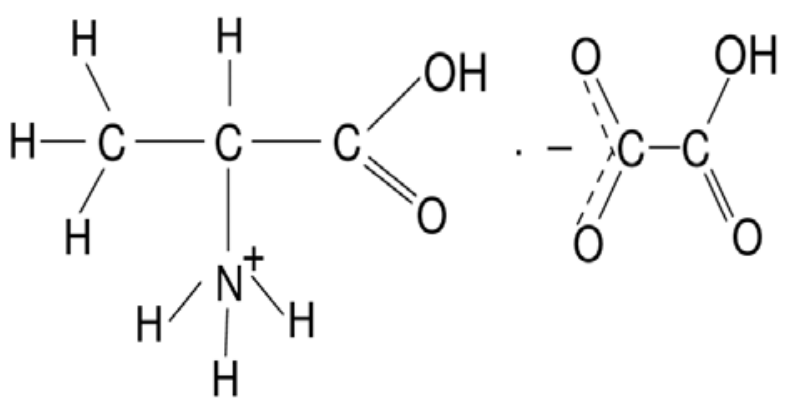

Fig. 4. Molecular structure of L-alaninium oxalate.

\subsection{Thermal Analysis}

The good thermal stability of LAO crystals up to $196^{\circ} \mathrm{C}$ establishes that it has prospects in laser applications where crystals should withstand high temperature. In the DTA curve (Fig. 5) there is a large endothermic peak at $196^{\circ} \mathrm{C}$ and a very small endothermic peak at around $98^{\circ} \mathrm{C}$. The major peak is the melting point of the crystal as the major weight loss occurs after that point and the minor peak is assumed to be a weak solid to solid phase transition as there is no weight loss at this temperature and there is no water of crystallization in the crystal. 


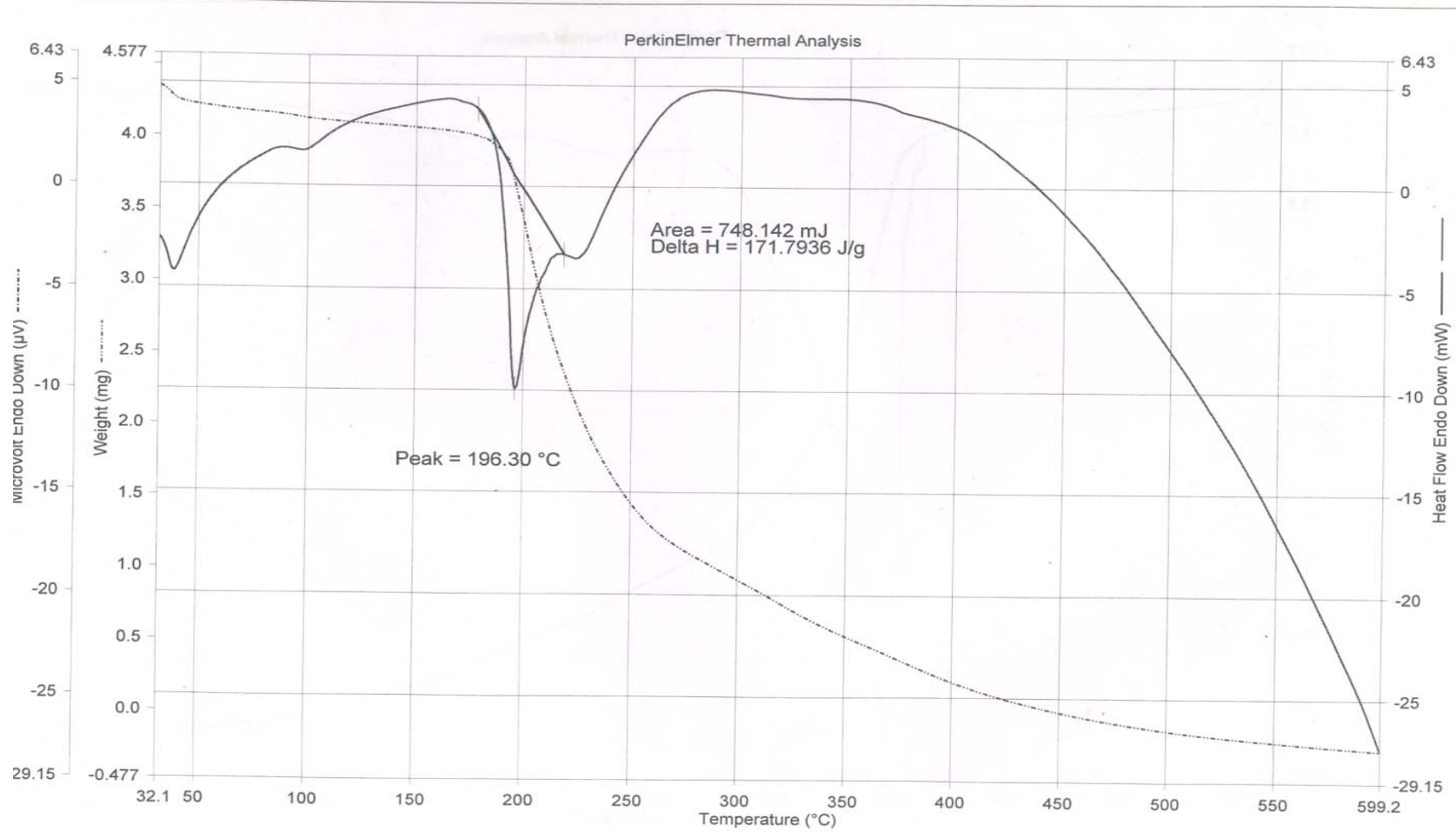

Fig. 5 TGA/DTA thermogram of L-alaninium oxalate

The differential scanning calorimetry (DSC) spectrum (Fig. 6) shows a minor endothermic peak at $98^{\circ} \mathrm{C}$ and a major peak at $197^{\circ} \mathrm{C}$, supporting the observation found in TGA/DTA measurement. In organic crystals phase transitions between polymorphs are very common and the mechanisms remain unknown despite of extensive investigations. No such detailed phase transition studies are reported in the literature for LAO, even though results are available for other organic crystals $[11,12]$. Hashizume et al [13] have observed the phase transition of Lethyl-3-urea by means of detailed temperature resolved single crystal diffraction method. Crystal structures before and after the phase transition are isostructural where one-dimensional hydrogen bonding structure is formed and stacked to form a molecular layer. Their work shows a solid to solid phase transition at $90^{\circ} \mathrm{C}$. Since these crystals are grown by slow evaporation method, they have taken care in omitting the occluded water, if any, during the growth by annealing the samples fairly at higher temperatures. In the present study LAO shows a phase transition (solid to solid) at $371 \mathrm{~K}\left(98^{\circ} \mathrm{C}\right)$, when the temperature is slowly increased from room temperature, which is of the same order as for L-ethyl-3-urea. The comparison with L-ethyl-3-urea is made here because both of them have layered structures. The layer structure of the present LAO, 
viewed from the $b$ axis is shown in Figure 3. Even though the geometry of the layers is retained, the relative positions of the layers in LAO with their neighbors change gradually with temperature. The change is accelerated at the temperature representing the start of the endotherm in the TGA and DTA curves. The structural variation thus creates a void space in between the layers and as this grows, naturally the crystal will be unstable and so the carboxyl group of the molecules turns into a disordered structure with abrupt conformational changes to fill up the void space. This transition, being isostructural, may be visualized as the transition of two elementary processes - supramolecular and molecular.

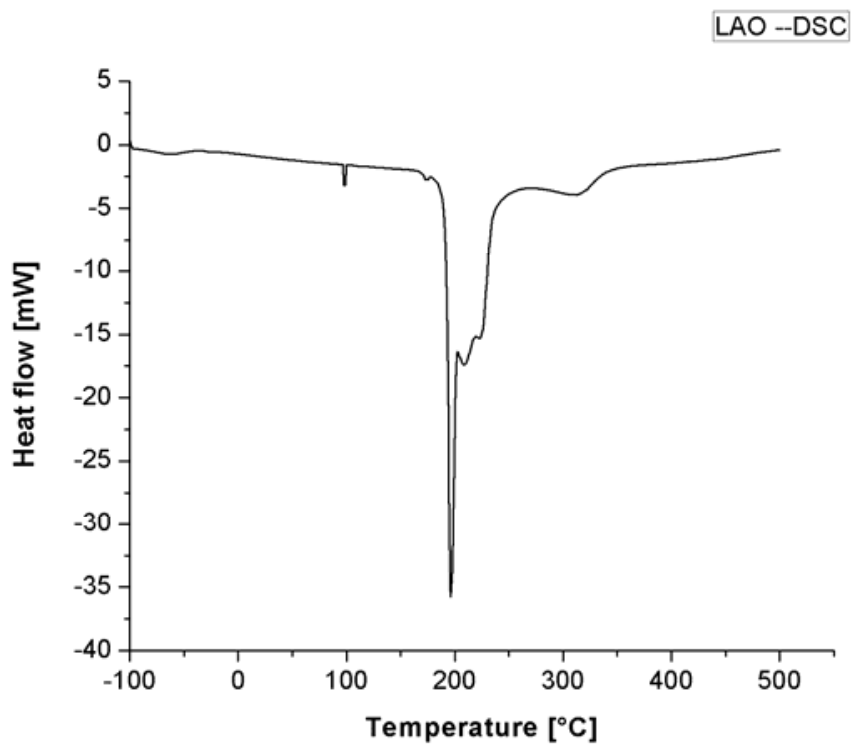

Fig. 6. Differential scanning calorimetry curve of L-alaninium oxalate

\subsection{Fourier Transform Infrared Spectroscopic Studies}

The characteristic vibrations of the carboxylate ions and the zwitter ionic group $\mathrm{NH}_{3}{ }^{+}$of $\mathrm{LAO}$ are depicted in the spectrum [Fig. 7]. Observed frequencies are compared with those of similar functional groups including carboxylate ions. During the formation of the salt; $\mathrm{NH}_{2}$ group in the free acid is converted into $\mathrm{NH}_{3}{ }^{+}$ions. In the spectrum, the peak at $3243 \mathrm{~cm}^{-1}$ corresponds to $\mathrm{OH}$ stretching of $\mathrm{COOH}$ group of amino acid; the one at $2900 \mathrm{~cm}^{-1}$ to $\mathrm{NH}_{3}^{+}$symmetric stretching in plane and the peak at $2509 \mathrm{~cm}^{-1}$ to $\mathrm{NH}_{3}{ }^{+}$symmetric stretch out of plane vibrations. The C-C overtone vibration is observed at $1915 \mathrm{~cm}^{-1}$, and $\mathrm{C}=\mathrm{C}$ stretching at $1716 \mathrm{~cm}^{-1}$. The vibration peak at1585 $\mathrm{cm}^{-1}$ is due to $\mathrm{NH}_{3}{ }^{+}$asymmetric bending and that at $707 \mathrm{~cm}^{-1}$ corresponds to $\mathrm{C}=\mathrm{O}$ bending $[14,15]$. The FTIR vibrational spectrum establishes the presence of $\mathrm{NH}_{3}{ }^{+}$group in the crystal confirming the protonation of amino acid group leading to the formation of LAO molecule [16]. 


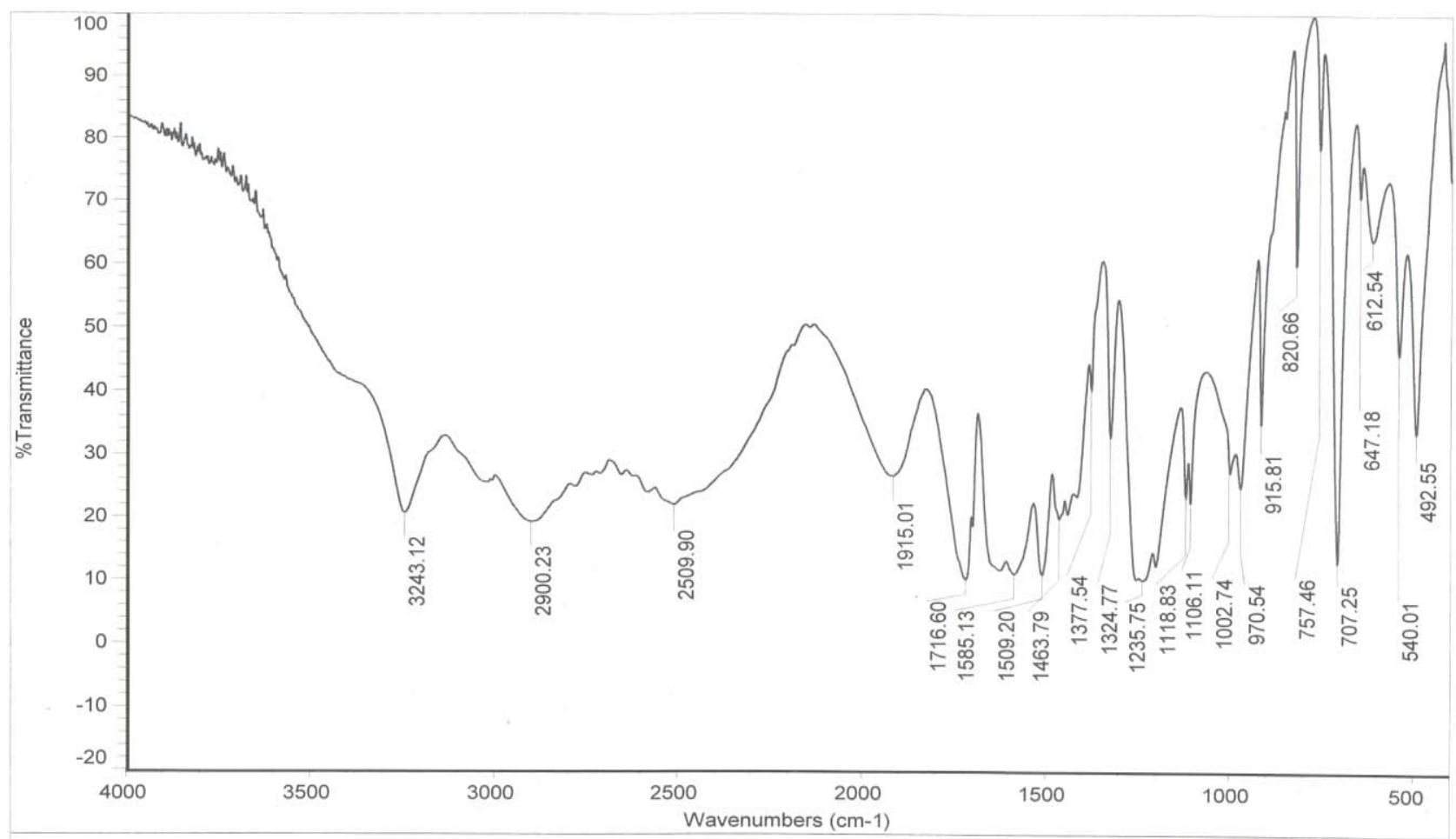

Fig. 7. FTIR spectrum of L-alaninium oxalate

\subsection{UV/ Vis/ NIR Absorption Spectroscopy}

UV/Vis/NIR absorption spectrum [Fig. 8] shows that LAO crystal has a wide transparency window without any absorption in the UV, visible and near IR regions, ranging from $318 \mathrm{~nm}$ to $1524 \mathrm{~nm}$ suggesting its suitability for second and third harmonic generations of the $1064 \mathrm{~nm}$ radiation and other applications in the blue - violet region.

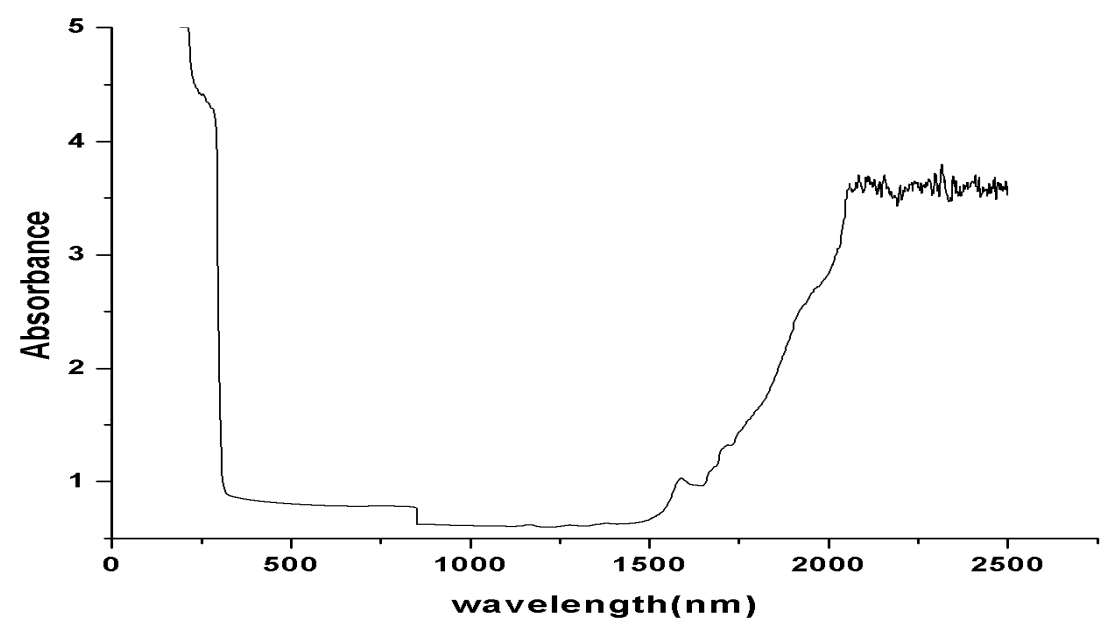

Fig. 8. UV/Vis/NIR absorption spectrum of L-alaninium oxalate 


\subsection{Photoconductivity Studies}

Field dependence of dark and photo currents of LAO crystals is shown in Figure 9. The photocurrent is found to be less than the dark current at every applied electric field. This phenomenon is known as negative photoconductivity which in this case may be due to the reduction in the number of charge carriers or their lifetime in the presence of radiation. Decrease in lifetime with illumination, could be due to the trapping process and increase in carrier velocity according to the relation:

$$
\tau=(\mathrm{vSN})^{-1}
$$

Where $\mathrm{v}$ is the thermal velocity of the carriers, $\mathrm{s}$ is the capture cross section of the recombination centers and $\mathrm{N}$ is the carrier concentration. As intense light falls on the sample, the lifetime decreases [17]. In Stockmann model, a two level scheme is proposed to explain negative photoconductivity [18]. The upper energy level is situated between the Fermi level and the conduction band, whereas the other one is located in the neighborhood of the valence band. The lower level has high capture cross section for electrons from the conduction band and holes from the valence band. As a result, as soon as the sample is kept under exposure to light, the recombination of electrons and holes takes place, resulting in decrease in the number of mobile charge carriers, giving rise to negative photoconductivity.

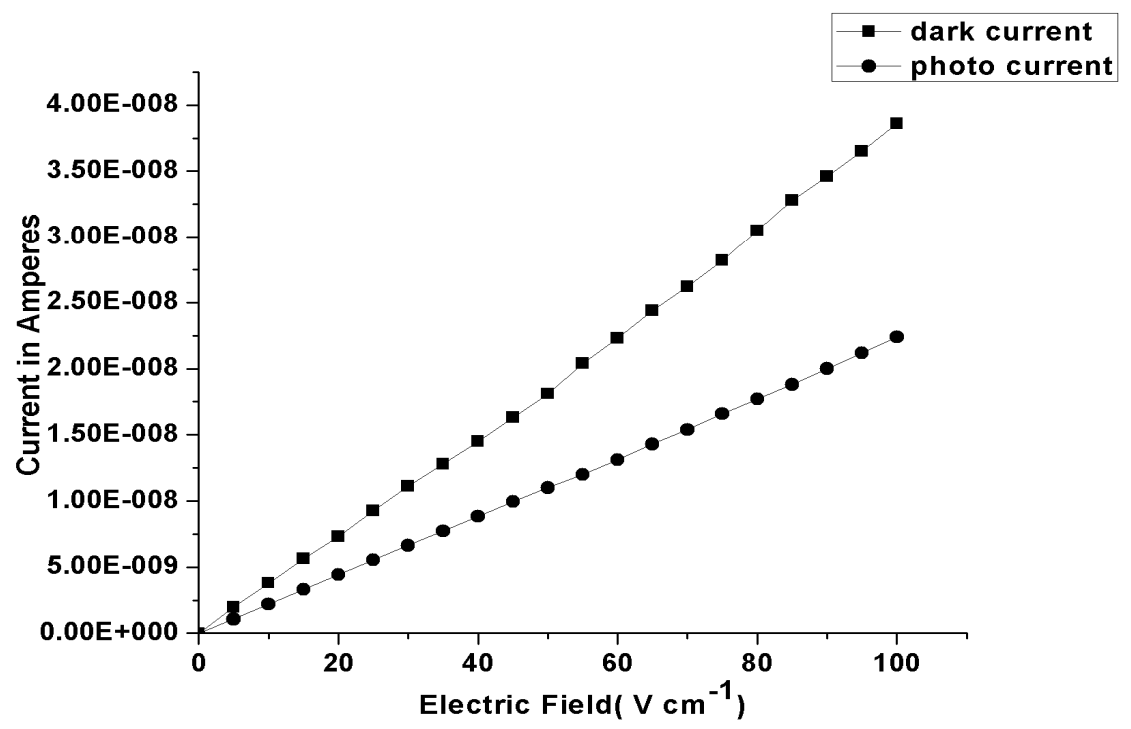

Fig. 9. Photoconductivity curve of L-alaninium oxalate crystal 


\subsection{Photoluminescence Studies}

Photoluminescence (PL) spectrum of LAO crystal recorded is shown in Figure 10. Excitation wavelength used is $300 \mathrm{~nm}$. Spectrum shows a broad peak centered at $440 \mathrm{~nm}$ with intensity comparable to that of conducting polymers and polymer composites. Intensity is slowly reduced in the higher wavelength region. The reason for enhanced PL emission in the case of LAO would be the presence of electron donating group $\mathrm{NH}$ and electron-withdrawing group $\mathrm{COOH}$ that can enhance the mobility of $\pi$ electrons. The maximum intensity peak at $440 \mathrm{~nm}$ is due to the protonation of amino group to the carboxyl group. The lowering of photoluminescence intensity at higher wavelength region may be attributed to a relatively low barrier for rotation of the carboxyl group around the central C-C bond [19].

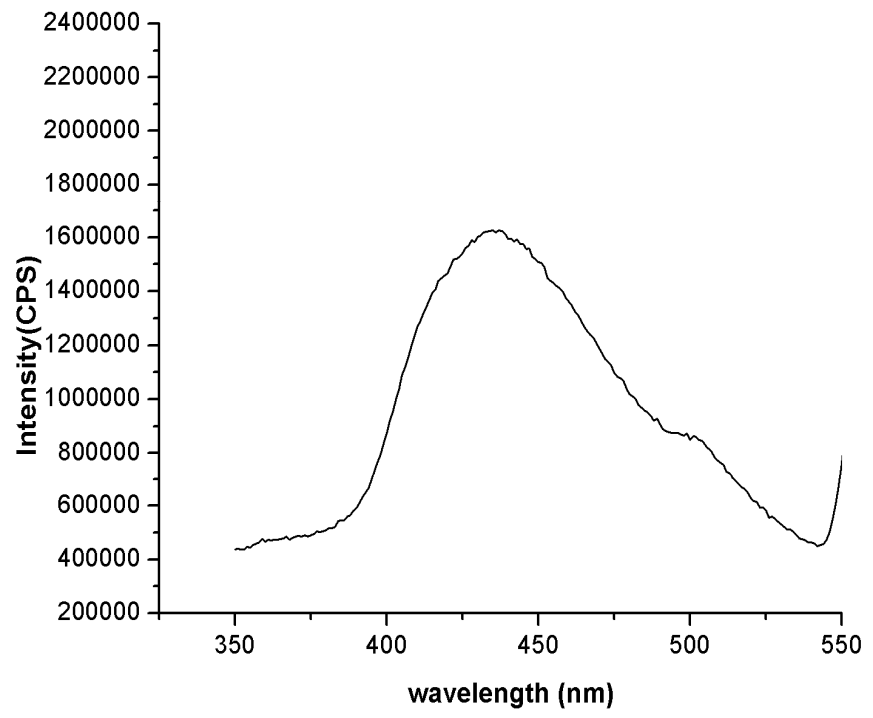

Fig. 10. Photoluminescence spectrum of L-alaninium oxalate

\subsection{Open Aperture Z Scan Measurements}

The result of the open aperture $Z$ scan measurement of the crystal is shown in Figure 11 . The open aperture (OA) curve demonstrates a nonlinear absorption and the characteristic pattern of the curve shows that the nonlinear absorption is reverse saturation absorption (RSA). For 532nm resonant absorption, both excited state absorption and two-photon absorption (TPA) can be responsible for the observed NLO effects. The RSA coefficient $\beta(\mathrm{m} / \mathrm{W})$ can be obtained from a best fitting performed on the experimental data of the OA measurement with the equations (1) and (2)[20] where $\alpha$ and $\beta$ are the linear and effective third order NLO absorption coefficients, respectively, $\tau$ is the time, $\mathrm{I}(\mathrm{z})$ is the irradiance and $\mathrm{L}$ is the optical path length. 


$$
\begin{aligned}
& \mathrm{T}(\mathrm{Z})=\frac{1}{Q(2) \sqrt{\pi}} \int_{-\infty}^{+\infty} \ln [1+Q(\sigma)] e^{-72} \mathrm{~d} \tau \\
& \mathrm{Q}(\mathrm{Z})=\beta \mathrm{I}(\mathrm{Z}) \frac{\mathrm{i-a}-\alpha 2}{\mathrm{a}}
\end{aligned}
$$

The value of the non linear absorption coefficient $\beta$ is calculated to be equal to be $2.9 \times 10^{-10}$ $\mathrm{m} / \mathrm{W}$. The reason for the large value of RSA coefficient may be due to the large number of delocalized $\pi$ electrons resulting from the protonation of amino group which is clear from the structure of the crystal (Fig.3). This delocalization gradually enhances the hyperpolarizability and the nonlinear susceptibility, and leads to large third-order NLO properties [21].

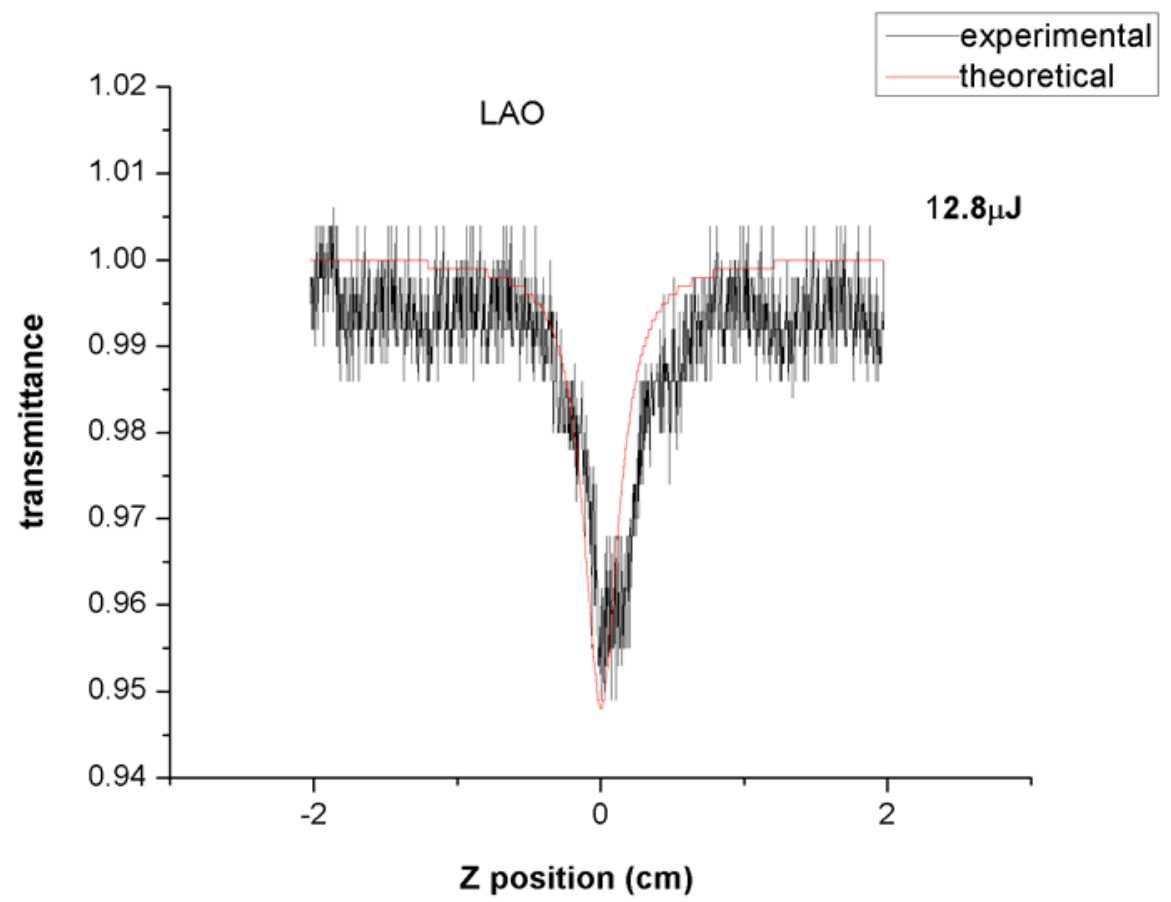

Fig. 11. Open aperture Z scan spectrum of L-alaninium oxalate crystal

\section{CONCLUSIONS}

The bulk size single crystals of L-alanininium oxalate are successfully grown by the slow evaporation solution growth method at room temperature. Exposure of the crystal surfaces to humid and dry atmospheres indicates that LAO is stable and non hygroscopic. The grown crystals have been subjected to various characterization studies. Crystal structure has been 
confirmed by powder XRD .CHN analysis reveals that the crystal doesn't contain any water of crystallization in its structure and suggests the molecular formula to be $\mathrm{C}_{5} \mathrm{H}_{9} \mathrm{NO}_{6}$. TGA/DTA and DSC studies establishes the good thermal stability of the crystal upto its melting temperature $\left(197^{\circ} \mathrm{C}\right)$, suggesting it to be a potential material for laser applications where crystal should withstand high temperatures. Since there is no decomposition observed up to $200^{\circ} \mathrm{C}$, crystallization can be done by melt method too.

FTIR studies confirm the various functional groups and their vibrational interactions. The UV/Vis/NIR absorption studies highlight the excellent transparency of the material in the range $318 \mathrm{~nm}$ to $1524 \mathrm{~nm}$. It is an important requirement for materials for applications in second and third harmonic generations. Open aperture $\mathrm{Z}$ scan studies establish that the material can be effectively used for optical limiting applications.

\section{References}

[1] M.D Aggarwal, J.Stephens, J.optoelectron.Adv.Mater, 5, 3 (2003).

[2] Razzetti.C, Ardoino.M, Zanotti, L.Zha, M.; Paorici.C, Cryst.Res.Technol, 37,456 (2002)

[3] McArdle. B. J, Sherwood .J. N, Damask A. C, J. Cryst. Growth, 22,193 (1974)

[4] Hampton. E. M, Shah. B. S, Sherwood. J. N, J. Cryst. Growth, 22, 22 (1974)

[5]S. Dhaushkodi, K.Vasantha, Cryst.Res.Technol, 39, 3 (2004)

[6] Bhat. H. L, Bull.Mater.sci, 17, 123 (1994).

[7] Marder. S. R, Sohn J. E, Stucky. G. D, Materials for Non-Linear Optics; American Chemical Society: Washington, DC, 1991.

[8] Arun.K.J, S.Jayalekshmi, Proceedings of the International conference on optoelectronic materials and thin films, CUSAT, Kochi, India, 2005.

[9]. M. Subha Nandhini, R. V.Krishnakumar, S.Natarajan, Acta Cryst. E57, 633 (2001).

[10] Ioffe. A. F, Phys. Status Solidi, 116, 457 (1989).

[11] Preethi Menon C and Philip J. Mater. Res. Bull. 36, 2407 (2001).

[12] Pichon.C, Appl. Phys. Lett. 35, 1435 (1979)

[13] Hashizhume.D, Acta Crystallogr. B59, 404, (2003).

[14] S.Ramasamy, R.K.Rajaram, J.Raman.Spectrosc.33, 689 (2002).

[15] L.Santra, A.L.Verma, J.Phys.Chem.solids, 55, 405 (1994)

[16] Arun.K.J, S.Jayalekshmi, AIP conf.proc, 1075, 115 (2008)

[17] V. N Joshi, Photoconductivity, Marcel Dekker, New York, (1990).

[18] R. H. Bube, Photoconductivity of solids, Wiley, New York, (1981).

[19] A. Aravindan, P. Srinivasan, Cryst. Res. Technol, 11, 1097 (2007)

[20] M. Sheik Bahae, A. A. Said, H. Wei, D. J. Hagan, IEEE J. Quantum. Electron, 26, 760 (1990).

[21] Arun.K.J, S.Jayalekshmi, Optoelectron. Adv. Mater (RC), Vol. 2, No. 11,701 (2008). 